This PDF is a selection from an out-of-print volume from the National Bureau of Economic Research

Volume Title: Evaluation of Econometric Models

Volume Author/Editor: Jan Kmenta and James B. Ramsey, eds.

Volume Publisher: Academic Press

Volume ISBN: 978-0-12-416550-2

Volume URL: http://www.nber.org/books/kmen80-1

Publication Date: 1980

Chapter Title: Comparison of Econometric Models by Optimal Control Techniques

Chapter Author: Gregory C. Chow

Chapter URL: http://www.nber.org/chapters/c11703

Chapter pages in book: (p. 229 - 243) 


\title{
Comparison of Econometric Models by Optimal Control Techniques
}

\author{
GREGORYC. CHOW \\ DEPARTMENT OF ECONOMICS \\ PRINCETON UNIVERSITY \\ PRINCETON, NEW JERSEY
}

An econometric model is ordinarily a system of simultaneous, stochastic difference equations involving endogenous variables, exogenous variables, policy variables, and parameters. It has many numerical characteristics. To characterize an econometric model or to compare two econometric models is a complicated task because of the many dimensions involved. Which characteristics are important depend on the particular use of the model. The purpose may be the estimation of certain structural parameters, the explanation of various aspects of business cycles, long-term or short-term forecasting, or policy analysis. In this paper, we will first summarize the existing, well-known characterizations of an econometric model (Section 1). We will then discuss two sets of techniques to describe the properties of a model. One is based on the theory of optimal control for deterministic systems (Section 2) and the other on the theory of optimal control for stochastic systems (Section 3). An illustration of the techniques of Section 3 using the Michigan Quarterly Econometric Model is given in Section 4. It should be pointed out at the outset that the word "comparison" in the title of this paper means "bringing out the important characteristics of" and does not mean comparative evaluation or deciding which model is better. The latter subject is discussed in Chow (1980). 


\section{Existing Characterizations of an Econometric Model}

It will be convenient for our discussion to start with a static system of simultaneous equations and then treat a dynamic system of simultaneous difference equations, leaving the problem of characterizing a dynamic stochastic system as the last topic.

\subsection{Static Model}

Both general and partial equilibrium models are systems of simultaneous equations. Two types of characteristics are of interest for such systems. First are the characteristics of individual equations, as summarized by their parameters, such as the elasticity of demand or the marginal propensity to consume. Second are the properties of the solution to the system. These are the properties of the reduced-form equations of an econometric model. Rather than characterizing the relationships among endogenous variables, such as the elasticity of demand and the marginal propensity to consume, they describe the responses of the solution values of the endogenous variables to changes in the exogenous variables and/or the parameters in the system. They describe the "comparative statics" induced by the system, and they contain the "multipliers" of an econometric model.

\subsection{Dynamic Deterministic Model}

Once the model becomes dynamic, being a system of difference or differential equations, its characteristics will be more complicated but can still be divided into two types. The characteristics of individual equations include short-run, intermediate-run, and long-run relationships among the variables in an equation, such as the short-run and long-run elasticities of demand and the short-run and long-run marginal propensities to consume. The long-run characteristics are the characteristics of the solution paths of the system, and they represent the effects of the exogenous variables on the solution paths. The solution paths may be damped or explosive; they may oscillate in various ways. The effects of the exogenous variables on the solutions are the subject of comparative dynamics. These effects are partly described by the various dynamic multipliers, including the impact multipliers, delayed multipliers (measuring the effects of a change in an exogenous variable in one period on the endogenous variables in a later period), and intermediate-run and longrun multipliers (measuring the cumulative effects of persistent changes in an exogenous variable for several or many periods on the current endogenous 
variables). The number of different dynamic multipliers is large because the time dimension is added into the picture. These multipliers are the coefficients of the "final form" of an econometric model which expresses the endogenous variables as functions of the exogenous variables after the lagged endogenous variables have been eliminated.

\subsection{Dynamic Stochastic Model}

After incorporating stochastic disturbances into an econometric model, we need further tools to characterize the stochastic solution paths of an econometric model. The first two moments of the solution path are of particular interest. The mean solution paths can be treated in the same way as in Section 1.2; all the dynamic multipliers and the comparative dynamic analyses are applicable to the mean paths. The variances and covariances of the endogenous variables may not be constant through time. If the time series generated by a system of stochastic difference equations are covariancestationary or nearly so, one can use the autocovariance matrix or the spectral density matrix to summarize many of the cyclical properties, as described, for example, in Chow (1975). Dynamic relationships between several variables can be summarized by cross-spectral densities and by observing the leads and lags between their turning points, in the same way that cycles of individual endogenous variables can be described by the spectral density functions and by the time intervals between turning points.

In this brief discussion of the existing tools to characterize econometric models, we have mentioned the use of structural parameters, reduced-form parameters and final-form parameters, and the tools to study cyclical properties of an econometric model. For any econometric model we can also derive the variances and covariances of the errors of its forecasts of the future values of the endogenous variables, given projected values of the exogenous variables. Thus models can be characterized and compared by the variances and covariances of the disturbances in the structural equations, in the reduced-form equations (measuring errors of forecasts one period ahead), and in the final-form equations (measuring errors of forecasts many periods ahead). For example, for an autoregressive model

$$
y_{t}=a y_{t-1}+u_{t}=a^{2} y_{t-2}+u_{t}+a u_{t-1}
$$

the error of a two period ahead forecast has a variance equal to $\operatorname{var}\left(u_{t}+\right.$ $\left.a u_{t-1}\right)$. One can also measure forecasting errors of an econometric model by comparing its various forecasts with actual historical observations. The remainder of this paper will be concerned with the characterization of an econometric model for the purpose of formulating an optimal economic policy. 


\section{Characterization of an Econometric Model by Deterministic Control}

In the optimal control of a deterministic econometric model (with its random disturbances set equal to their expected values), a multiperiod loss function is postulated, and its value is minimized with respect to the time paths of the policy or control variables (a subset of exogenous variables), subject to the constraint of the dynamic model. If the econometrician is willing to choose a loss function, then models can be characterized and compared by the solution paths of the key endogenous and policy variables. Such comparisons have recently been made by the U.S. econometric model builders who were participants in an NSF-NBER Seminar on Econometric Model Comparison under the chairmanship of Lawrence Klein. Some of the results are described in Hirsch, Hymans, \& Shapiro (1978) and in Chow \& Megdal (1978a). Optimal deterministic control paths were obtained from several models of the U.S. economy for the 17 quarters from 1971.1 to 1975.1 using the same loss function which penalizes the squared deviations of the inflation rate, unemployment rates, real GNP, and balance of trade from their preassigned targets. To illustrate the results, the optimal solutions for the inflation rate show whether or not the two-digit inflation in 1974 could have been avoided by suitable economic policy beginning in 1971.1 according to the participating models. The answer is mostly negative. The solutions for the unemployment rate by and large show that significant reductions could have been achieved during quarters of high unemployment without seriously aggravating the inflation situation. Note that this solution does not take into account the possible delayed effects of an expansionary policy on inflation after 1975.1, which was the terminal quarter of the multiperiod optimization problem.

Similarly, the optimal solution paths for the policy variables can also be compared to show similarities and differences among models. In the experiments conducted by the participants of the Econometric Model Comparison Seminar, the policy variables are federal government nondefense expenditures and unborrowed reserves. The optimal solutions for government expenditures according to several models require fairly sizable increases, in the order of 50 to 60 billions (at an annual rate) in the later quarters over the actual expenditures. One can further compare the relative roles played by the fiscal and monetary instruments across different models. Not only the average deviations of the optimal settings of these instruments from some norms (such as historical trends) but also their relative fluctuations through time can be compared. In short, different characteristics of the optimal 
solution paths for the important endogenous and policy variables in a deterministic control problem can be compared among different econometric models.

A natural extension of the above comparison is to vary the parameters in the loss function and to observe the resulting changes in the optimal solution paths. This is an application of the method of comparative dynamics to deterministic models. In fact, it has been suggested by Chow \& Megdal (1978b) that the parameters in a quadratic loss function should be varied in a systematic way in order to trace out the best available trade-off possibilities for unemployment and inflation implicit in an econometric model. Econometricians have attempted to derive the trade-off relationships between unemployment and inflation from an econometric model by recording the behavior of these two variables in simulations using more or less expansionary, but still fairly arbitrary, paths for the policy instruments. Simulations of this type have been performed by Anderson \& Carlson (1972), de Menil \& Enzler (1972), Hirsch (1972), Bodkin (1972), and Hymans (1972), among others. This method is defective because the unemployment and inflation rates so obtained without optimization could usually beimproved upon, as demonstrated by the calculations using the St. Louis Model and the Michigan Quarterly Econometric Model reported in Chow \& Megdal (1978b).

In order to determine the lowest inflation rate corresponding to a $6 \%$ unemployment rate, we solve an optimal control problem using a quadratic loss function with $1 \%$ and $6 \%$ as the targets for the inflation and unemployment rates, respectively (assuming a $1 \%$ annual inflation rate to be lower than achievable), and 1 and 100 , respectively, as the weights penalizing the squared deviations of inflation and unemployment from their targets. Optimization will ensure that the unemployment rate is close to $6 \%$ and the inflation rate will be made as low as possible. Here we are dealing with a multiperiod optimization problem. The inflation rates obtained from the optimal solution will change from quarter to quarter, but the entire set of inflation rates could not be improved upon in the sense that, given a $6 \%$ unemployment rate, the sum of squared deviations of the inflation rates in all periods from $1 \%$ is the minimum. If one wishes to depict in a two-dimensional diagram the trade-off possibilities between inflation and unemployment for many periods, then the mean rates of these variables over time or their root mean squared deviations over time could be plotted. The points in the diagram are obtained by solving several optimal deterministic control problems as formulated above, with the target for the unemployment rate varying from $4 \%$ to $9 \%$ or over whatever range of values required. Thus each econometric model is characterized by one optimal unemployment-inflation trade-off curve. This curve permits us to answer the important question concerning 
the model: Can a $4 \%$ inflation rate be achieved while maintaining an unemployment rate of $5 \%$ during a particular time period?

Before closing this section, we would like to describe briefly some of the available algorithms to calculate the solutions to deterministic control problems using an econometric model. The algorithms can be divided into two categories. The first treats the problem strictly as a deterministic control problem, ignoring all the random elements in the econometric model. Since the time paths of the endogenous variables are determined by the time paths of the control variables through the econometric model, and the multiperiod loss is a function of the endogenous (and possibly also the control) variables, one can regard the loss function as a function of the control variables. Various gradient-type algorithms have been applied to minimize the loss function with respect to the time paths of the control variables, including the works of Fair (1974), Holbrook (1974), Craine, Havenner, \& Tinsley (1976), Kalchbrenner \& Tinsley (1976), Ando \& Palash (1976), and Norman, Norman \& Palash (1975). The second category is a by-product of a solution to an optimal stochastic control problem which allows for random disturbances in an econometric model. The mathematical expectation of a multiperiod loss function is minimized subject to the constraint of a stochastic econometric model, as will be illustrated by (3.2) and (3.3) of Section 3. The optimal solution will take the form of feedback control equations, i.e., $x_{t}=G_{t} y_{t-1}+g_{t}$, where $x_{t}$ and $y_{t}$ are respectively vectors of control and endogenous variables, and $G_{t}$ and $g_{t}$ are respectively a matrix and a vector of constants to be determined by the optimal control algorithm. To obtain a solution to a deterministic control problem, however, we can apply these optimal feedback control equations to a deterministic econometric model and calculate the time paths of both the endogenous and the control variables when the model is subject to optimal control.

One method to obtain a set of optimal feedback control equations to minimize a quadratic loss function subject to a system of nonlinear structural equations as described in Chow $(1975$, p.285,1976) is the following. First, tentative paths $x_{t}^{0}$ of the control variables are chosen. The Gauss-Siedel method is applied to solve the nonlinear equations to obtain the paths $y_{t}^{0}$ of the endogenous variables corresponding to the chosen paths of the control variables. The econometric model is linearized about these paths:

$$
\begin{aligned}
y_{t}= & \phi\left(y_{t}, y_{t-1}, x_{t}\right) \simeq \phi\left(y_{t}^{0}, y_{t-1}^{0}, x_{t}^{0}\right)+\Phi_{1 t}\left(y_{t}-y_{t}^{0}\right) \\
& +\Phi_{2 t}\left(y_{t-1}-y_{t-1}^{0}\right)+\Phi_{3 t}\left(x_{t}-x_{t}^{0}\right),
\end{aligned}
$$

where $\Phi_{i t}$ denotes the matrix of the derivatives of the vector function $\phi$ with respect to its $i$ th argument evaluated at $y_{t}^{0}, y_{t-1}^{0}$, and $x_{t}^{0}$. A set of time-varying, linear structural equations is thus obtained. These equations are solved to 
produce a set of time-varying, linear reduced-form equations. Given these reduced-form equations and the quadratic loss function, the solution to the deterministic optimal control problem is computed in the form of optimal feedback control equations. These equations, together with the original nonlinear structural equations, determine the time paths of the endogenous and control variables when the system is governed by the new set of control rules. The model is again linearized about these paths as before. The process continues until the solution paths from successive linearizations converge.

\section{Char acterization of an Econometric Model by Stochastic Control}

The feedback control algorithm described at the end of Section 2 provides a nearly optimal solution to the original stochastic control problem when the random disturbances in the econometric model are retained. It can therefore be used to characterize a stochastic econometric model from the viewpoint of optimal economic policy. When the random disturbances of an econometric model are taken into account in the formulation of economic policy, two important consequences should be noted. First, as we have pointed out, the solution will take the form of feedback control equations. Second, the solution paths for both endogenous and control variables become stochastic. In order to characterize them, we need at least the expected paths and the autocovariance matrices of the variables in the system under feedback control. The expected paths can be approximated by the solution to the deterministic control problem obtained by replacing the random disturbances by their expected values. The autocovariance matrix of the system can be easily computed by using the time-varying, linear feedback control equations and the linearized reduced-form equations obtained from the last iteration (or linearization) using the preceding algorithm. See Chow (1975, Chapter 3), for example, on the methods of computing the autocovariance matrix. Hence the dynamic properties of the optimal control paths of the endogenous and control variables can be used to characterize and compare stochastic econometric models as well as deterministic models.

When dealing with stochastic models, we can supplement the unemployment-inflation trade-off curves proposed in Section 2. These curves are constructed from the deterministic time paths of these variables in the optimal deterministic control solution or from the expected time paths, denoted by $\bar{y}_{t}$, of the (approximately) optimal stochastic control solution. Let $y_{1 t}$ and $y_{2 t}$ stand for the unemployment and inflation rates respectively. We could vary the weights in the loss function, as suggested in Section 2, and plot the 
resulting combinations of $\sum_{t=1}^{T} \bar{y}_{1 t} / T$ and $\sum_{t=1}^{T} \bar{y}_{2 t} / T$. We could also plot the square roots of $\sum_{t=1}^{T}\left(\bar{y}_{1 t}-a_{1 t}\right)^{2} / T$ and $\sum_{t=1}^{T}\left(\bar{y}_{2 t}-a_{2 t}\right)^{2} / T$, where $a_{1 t}$ and $a_{2 t}$ are some target paths for unemployment and inflation and may be set equal to zero. The contribution of the $i$ th variable to the multiperiod expected loss is given by

$$
\sum_{i=1}^{T} E\left(y_{i t}-a_{i t}\right)^{2}=\sum_{t=1}^{T}\left(\bar{y}_{i t}-a_{i t}\right)^{2}+\sum_{t=1}^{T} E\left(y_{i t}-\bar{y}_{i t}\right)^{2} .
$$

Hence the sums $\sum_{i=1}^{T} E\left(y_{i t}-\bar{y}_{i t}\right)^{2}$ of the variances over $T$ periods for the unemployment rate and the inflation rate can be plotted. Or the total contributions, consisting of $\sum_{t=1}^{T}\left(\bar{y}_{i t}-a_{i t}\right)^{2}$ and $\sum_{t=1}^{T} E\left(y_{i t}-\bar{y}_{i t}\right)^{2}$, from the unemployment and inflation rates can be plotted on a two-dimensional diagram. A curve can be traced out by varying the parameters of the loss function as the unemployment-inflation trade-off curve was traced out in Section 2.

The main purpose of this section, however, is to propose some summary measures to characterize an econometric model for policy purpose, rather than simply exhibiting the mean paths and the covariance matrix of the major economic variables prevailing when the model is subject to optimal feedback control. The basic idea is a generalization of the reduced-form equations of a static model relating the endogenous variables to the control variables. The multipliers are the derivatives of these reduced-form equations. Our problem is complicated because we are studying the effects of the policy variables in the context of a nonlinear, dynamic, stochastic econometric model, which is being controlled to minimize the expectation of a multiperiod objective function. Rather than measuring the effects on many individual endogenous variables, we propose to measure the effects of the current control variables on the total expected loss for all future periods until the end of the planning horizon. We will choose a scalar function relating the multiperiod expected loss to the control variables of the first period. This function is derived directly from the well-known stochastic control theory based on the method of dynamic programming as follows.

First, assume a quadratic loss function for $T$ periods with $k_{t}=K_{t} a_{t}$

$$
W=\sum_{t=1}^{T}\left(y_{t}-a_{t}\right)^{\prime} K_{t}\left(y_{t}-a_{t}\right)=\sum_{t=1}^{T}\left(y_{t}^{\prime} K_{t} y_{t}-2 y_{t}^{\prime} k_{t}+a_{t}^{\prime} K_{t} a_{t}\right) .
$$

Second, the nonlinear econometric model is linearized about the solution paths of the optimal deterministic control problem as described at the end of the last section, yielding the following reduced-form equations

$$
y_{t}=A_{t} y_{t-1}+C_{t} x_{t}+b_{t}+u_{t},
$$


where $x_{t}$ is a vector of control variables which may be incorporated as a subvector of the endogenous variables $y_{t}$ if necessary and $u_{t}$ is a vector of serially uncorrelated random disturbances. Using the method of dynamic programming (Chow, 1975, Sec. 8.1), we find the optimal strategy by first minimizing the expected loss $E\left(y_{T}-a_{T}\right)^{\prime} K_{T}\left(y_{T}-a_{T}\right)$ for only the last period $T$ with respect to $x_{T}$. Substituting (3.3) for $y_{T}$ in this expectation and differentiating the result with respect to $x_{T}$, we obtain a linear feedback control equation $x_{T}=G_{T} y_{T-1}+g_{T}$. We then minimize the sum of the expected losses for the last two periods with repsect to $x_{T-1}$, assuming that the last period policy $x_{T}$ shall be optimal, i.e., substuting the minimum expected loss for period $T$ into the minimand. Continuing the process backward in time, we finally minimize the sum of the expected losses for all $T$ periods with respect to $x_{1}$ of the first period, assuming that $x_{2}, \ldots, x_{T}$ will be optimal. This sum, after all the future minimum expected losses from period 2 onward have been duly inserted, is the expectation of a quadratic function of the economic variables $y_{1}$ for the first period only:

$$
V_{1}=E\left(y_{1}^{\prime} H_{1} y_{1}-2 y_{1}^{\prime} h_{1}+c_{1}\right)
$$

where the coefficients $H_{1}, h_{1}$, and $c_{1}$ can be calculated by standard formulas (Chow, 1975, p.179). Using (3.3) to substitute for $y_{1}$ in (3.4) and taking expectations, we have

$$
\begin{aligned}
V_{1}= & x_{1}^{\prime} C_{1}^{\prime} H_{1} C_{1} x_{1}+2 x_{1}^{\prime} C_{1}^{\prime}\left(H_{1} A_{1} y_{0}+H_{1} b_{1}-h_{1}\right) \\
& +\left(A_{1} y_{0}+b_{1}\right)^{\prime} H_{1}\left(A_{1} y_{0}+b_{1}\right)+E\left(u_{1}^{\prime} H_{1} u_{1}\right)-2\left(A_{1} y_{0}+b_{1}\right)^{\prime} h_{1}+c_{1} \\
= & x_{1}^{\prime} Q x_{1}+2 x_{1}^{\prime} q+d
\end{aligned}
$$

Thus, assuming that the policies from period 2 onward will be optimal, the expected multiperiod loss is a quadratic function of $x_{1}$ as given by (3.5). The optimal first-period policy $\hat{x}_{1}$ is obtained by minimizing (3.5) with respect to $x_{1}$, yielding the associated minimum expected multiperiod loss $\hat{V}_{1}=V_{1}\left(\hat{x}_{1}\right)$. Note that the subscript of $V$ denotes the fact that this cumulated expected loss is computed from period 1 onward.

Our proposal is to use the quadratic function $V_{1}\left(x_{1}\right)$, as given by (3.5), to characterize and compare econometric models. This function gives the total expected loss from period 1 to period $T$ in terms of the control variables $x_{1}$ in the first period, assuming that future policies from period 2 to period $T$ will be chosen optimally. It appears to capture the essential information contained in an econometric model concerning the effects of the current policy variables on economic welfare as measured by the loss function. It is applicable to deterministic models as a special case where the vectors $u_{t}$ of random disturbances for all periods are set equal to zero. The constant 
term $d$ in the quadratic function (3.5) will be affected since $E\left(u_{1}^{\prime} H_{1} u_{1}\right)$ is zero and its component $c_{1}$ is dependent on future $E\left(u_{t}^{\prime} H_{t} u_{t}\right)$.

To compare two econometric models $\mathrm{A}$ and $\mathrm{B}$, we use the functions $V_{1 \mathrm{~A}}\left(x_{1}\right)$ and $V_{1 \mathrm{~B}}\left(x_{1}\right)$ obtained by the dynamic programming algorithm applied to these models respectively, given the same multiperiod loss function. Let $\hat{x}_{1 \mathrm{~A}}$ and $\hat{x}_{1 \mathrm{~B}}$ respectively minimize $V_{1 \mathrm{~A}}$ and $V_{1 \mathrm{~B}}$. One can certainly compare the two models by their first-period optimal policies $\hat{x}_{1 \mathrm{~A}}$ and $\hat{x}_{1 \mathrm{~B}}$. In fact, in Section 2 we have pointed out that the entire optimal solution paths $\hat{x}_{t \mathrm{~A}}$ and $\hat{x}_{t \mathrm{~B}}(t=1, \ldots, T)$ obtained from two deterministic models can be compared. However, by simply inspecting the values of $\hat{x}_{1 \mathrm{~A}}$ and $\hat{x}_{1 \mathrm{~B}}$ we cannot tell how different the policy recommendations from the two models are. Perhaps the function $V_{1 \mathrm{~A}}\left(x_{1}\right)$ is fairly flat around $\hat{x}_{1 \mathrm{~A}}$, and $V_{1 \mathrm{~A}}\left(\hat{x}_{1 \mathrm{~B}}\right)$ is not much larger than $V_{1 \mathrm{~A}}\left(\hat{x}_{1 \mathrm{~A}}\right)$. This means that, as far as model $\mathrm{A}$ is concerned, $\hat{x}_{1 \mathrm{~B}}$ is about as good a policy as $\hat{x}_{1 \mathrm{~A}}$. On the other hand, $V_{1 \mathrm{~B}}\left(\hat{x}_{1 \mathrm{~A}}\right)$ may be much larger than $V_{1 B}\left(\hat{x}_{1 B}\right)$; that is, as far as model B is concerned, its optimal policy $\hat{x}_{1 B}$ is much superior to the optimal policy $\hat{x}_{1 A}$ derived from model A.

To depict the differences between two models $\mathrm{A}$ and $\mathrm{B}$ for the purpose of obtaining a reasonable first-period policy $x_{1}$, the following payoff matrix can be used as an example:

States of the World

A

B

Optimal strategy from A

Optimal strategy from B

\begin{tabular}{|ll|ll|}
\hline$V_{1 \mathrm{~A}}\left(\hat{x}_{1 \mathrm{~A}}\right)$ & 100 & $V_{1 \mathrm{~B}}\left(\hat{x}_{1 \mathrm{~A}}\right)$ & 95 \\
\hline$V_{1 \mathrm{~A}}\left(\hat{x}_{1 \mathrm{~B}}\right)$ & 300 & $V_{1 \mathrm{~B}}\left(\hat{x}_{1 \mathrm{~B}}\right)$ & 90 \\
\hline
\end{tabular}

When we consider the welfare consequence of using the optimal policy $\hat{x}_{1 \mathrm{~A}}$ from model $\mathrm{A}$ if model $\mathrm{B}$ happens to be true, we do not assume that the policy recommendations from model A will be followed period after period. Rather, we assume that the (mistaken) policy from model A will be followed only in the current period and the (correct) policies from the hypothetically true model B might be followed from period 2 on. The rationale for the above payoff matrix is that the decision maker is not committed to follow the mistaken policies from an incorrect model in the future. To measure the welfare loss in adopting $\hat{x}_{1 \mathrm{~A}}$ for only the first period while allowing for the possibility to behave optimally later on assuming model $\mathrm{B}$ to be true, we compare $V_{1 \mathrm{~B}}\left(\hat{x}_{1 \mathrm{~A}}\right)$ with $V_{1 \mathrm{~B}}\left(\hat{x}_{1 \mathrm{~B}}\right)$ in the above matrix. If $V_{1 \mathrm{~B}}\left(\hat{x}_{1 \mathrm{~A}}\right)-V_{1 \mathrm{~B}}\left(\hat{x}_{1 \mathrm{~B}}\right)$ is small while $V_{1 \mathrm{~A}}\left(\hat{x}_{1 \mathrm{~B}}\right)-V_{1 \mathrm{~A}}\left(\hat{x}_{1 \mathrm{~A}}\right)$ is large, as illustrated by the numbers given for the payoff matrix above, one would adopt $\hat{x}_{1 \mathrm{~A}}$ by the minimax criterion. $\mathrm{A}$ Bayesian would assign probabilities $P(\mathrm{~A})$ and $P(\mathrm{~B})$ to the two models and adopt the policy to minimize expected loss. In the above numerical illustra- 
tion, unless $P(\mathrm{~A})$ is very small as compared with $P(\mathrm{~B}), \hat{x}_{1 \mathrm{~A}}$ will yield a smaller expected loss than $\hat{x}_{1 \mathrm{~B}}$.

If model $A$ is assumed to be fairly accurate, we can evaluate a historically adopted policy $x_{1}$ by the difference $V_{1 \mathrm{~A}}\left(x_{1}\right)-V_{1 \mathrm{~A}}\left(\hat{x}_{1 \mathrm{~A}}\right)$, where the function $V_{1 \mathrm{~A}}(\cdot)$ is obtained by using the historical period in question as period 1 . This difference measures the welfare cost of adopting the historical policy $x_{1}$, rather than the optimal policy $\hat{x}_{1 \mathrm{~A}}$. The logic is identical with that of comparing $V_{1 \mathrm{~A}}\left(\hat{x}_{1 \mathrm{~B}}\right)$ and $V_{1 \mathrm{~A}}\left(\hat{x}_{1 \mathrm{~A}}\right)$ as suggested in the previous paragraph. This approach to the evaluation of historical policies is discussed in Chow (1978).

\section{An Illustration Using the Michigan Model}

To illustrate the method of Section 3, the Michigan Quarterly Econometric Model (Hymans \& Shapiro, 1973) has been used to calculate the function $V_{1}\left(x_{1}\right)$. The optimal control problem solved is the one posted by the participants of the NSF-NBER Econometric Model Comparison Seminar referred to in Section 2. That is, the number of periods is 17 , covering the quarters from 1971.1 to 1975.1 . The objective is to minimize the loss function

$$
\begin{aligned}
& \sum_{t=1}^{17}\left[\dot{p}_{t}-\alpha_{t}\right)^{2}+.75\left(u_{t}-4.0\right)^{2}+.75\left(\mathrm{GNP} \mathrm{gap}_{t}-0\right)^{2} \\
& \left.+\left(\mathrm{TB}_{t}-0\right)^{2}+.1\left(\mathrm{UR} \$_{t}-\gamma_{t}\right)^{2}\right],
\end{aligned}
$$

where $\dot{p}$ is the annual rate of inflation measured by the GNP deflator, $\alpha_{t}=3.0$ for $t=1, \ldots, 12, \alpha_{t}=7.0$ for $t=13, \ldots, 17, u$ is the unemployment rate, GNP gap is the percentage deviation of GNP in 1958 dollars (GNP58) from capacity output, TB is trade balance as a percentage of GNP in current dollars, URS is unborrowed reserves in billions of current dollars, and $\gamma_{t}$ represents a smooth expansionary path for UR\$. The policy variables set up for our computations are government transfer payments GTRP\$, unborrowed reserves UR $\$$, and nondefense government purchases of goods and services GFO\$, all in billions of current dollars. However, the first control variable GTRP $\$$ was not treated as such by the participants of the Econometric Model Comparison Seminar. Therefore, we simply fixed it as its historical path by using the historical path as the target path and assigning a large penalty weight of 100 to the square of its deviation from target [this part of the loss function not being shown in function (4.1)]. The UR\$ term in the loss function serves to prevent erratic behavior of the monetary instrument. The estimated residuals in the structural equations were added back to the intercepts, at the suggestion of the seminar participants, resulting in a deterministic control problem although our method can handle the stochastic 
case as well. The optimal control solution paths for the major endogenous and control variables have been discussed elsewhere (Chow \& Megdal, 1978a). We will study the function $V_{1}\left(x_{1}\right)$ below.

The quadratic function $V_{1}\left(x_{1}\right)=x_{1}^{\prime} Q x_{1}+2 x_{1}^{\prime} q+d$ is given by the following matrix $Q$ and vector $q$

$$
Q=\left[\begin{array}{rrr}
100.00097 & .00003 & .00237 \\
.00003 & .10301 & .00100 \\
.00237 & .00100 & .00861
\end{array}\right], \quad q=\left[\begin{array}{r}
-8270.316 \\
-2.798 \\
-0.897
\end{array}\right]
$$

The vector of control variables in period 1 which minimizes $V_{1}$ is

$$
\hat{x}_{1}^{\prime}=\left[\begin{array}{lll}
82.7005 & 26.3770 & 78.3233
\end{array}\right] \text {. }
$$

The derivatives of $V_{1}$ with respect to the three control variables are $2 Q x_{1}$ $+2 q$. They are of course zero at $x_{1}=\hat{x}_{1}$. If the first control variable $x_{11}$ deviates slightly from its minimizing value 82.70 , say $x_{11}=83.70$ billion, and the other two control variables retain their optimum values, the derivative of $V_{1}$ with respect to the first is very large, being equal to 100.0 , which is the leading diagonal element of $Q$. On the other hand, when the second and third control variables are increased by 1 billion from their optimum values, the derivatives will only be .1030 and .0086 , respectively. The function $V_{1}$ increases very sharply as $x_{11}$ deviates from its optimum value because we have put a heavy penalty on the deviation of this variable from its historical or target value 82.70 ; we are in fact not treating this variable as a genuine control variable. $V_{1}$ increases more rapidly when the second control variable (unborrowed reserves) deviates from its optimum than when the third control variable (nondefense government purchases) deviates from its optimum, partly because the former variable enters explicitly in the loss function (4.1).

Since the first control variable is really fixed, we will simplify our analysis by reducing $V_{1}$ to a function of only the second and third control variables. When $x_{11}$ is fixed at its historical value 82.70 , the quadratic function $V_{1}$ involves

$$
Q=\left[\begin{array}{ll}
.103,013 & .000,999 \\
.000,999 & .008,614
\end{array}\right], \quad q=\left[\begin{array}{l}
-2.7954 \\
-0.7011
\end{array}\right] .
$$

If we had computed the function $V_{1}$ for another econometric model, we would use its optimum values for the two control variables to evaluate the function (4.4) and compare the result with the minimum value of (4.4). We would also use the optimum values for (4.4) to evaluate the function $V_{1}$ derived from the other model and compare the result with the minimum of the latter function. We could also plot the contour maps of these two functions on the same diagram and compare them. Without the second $V_{1}$ at our disposal, we will 
evaluate (4.4) at the historical values of the control variables, which are 29.5 and 24.1, respectively. Note that the second figure differs a great deal from the optimum value 78.3 given in (4.3). At these historical values, the multiperiod loss $V_{1}$ given by (4.4) is 26.00 higher than its minimum.

To examine the figure 26.00 more closely, we will compute the difference between the first-period losses of the two policies. The figure 26.00 is composed of this difference and the remainder which measures the extra loss from period 2 to $T$ attributable to the historical (nonoptimal) policy as it affects the initial economic condition at the end of period 1 . The optimum solution values and the historical solution values of the variables entering the loss function (4.1) for period 1 (1971.1) are given in the accompanying tabulation.

\begin{tabular}{lcccrc}
\hline Solution & $\dot{p}_{1}$ & $u_{1}$ & GNP gap & TB $_{1}$ & UR\$ \\
\hline Optimum & 6.07 & 5.08 & .48 & -.182 & 26.4 \\
Historical & 4.69 & 5.95 & 5.01 & .278 & 29.5 \\
\hline
\end{tabular}

The first-period losses resulting from these two solutions are 11.47 and 24.61, respectively, the difference being 13.14 . (Note that the target value for UR $\$_{1}$ was set at its historical value 29.5 , accounting for a contribution of .1(26.4 $-29.5)^{2}=.961$ to the first-period loss of the optimum policy.) The main contribution to the first-period loss of the historical policy is from the GNP gap, equal to $.75(5.01)^{2}=18.83$. Although the loss function (4.1) weighs the inflation term by 1 and the unemployment term by only .75, it penalizes low output quite heavily through the GNP gap. In short, 13.14 or about half of the extra multiperiod loss 26.00 is allotted to the difference between the firstperiod losses. The assumption is that, no matter whether the first-period policy is optimal or not, the policies from period 2 on will be optimally chosen. The nonoptimal policy in period 1 adds 13.14 to the loss in period 1 itself, as computed from the two sets of solution values of the variables included in the loss function shown above. It adds an almost equal amount to the total loss from period 2 to period 17 , assuming the policies in these periods to be optimal. The 26.00 figure can also be compared with the total loss of 248.03 for all 17 periods if optimal policies had been followed throughout.

The fairly sizable difference between the outcomes of the two policies is not surprising because the historical value of federal government nondefense purchases in 1971.1 is 24.1 billion and its optimal value from solving the optimal control problem using the loss function (4.1) is 78.3 billion. We have not considered the political feasibility of such a large increase in government expenditures. To do so would require putting an extra term for this control variables in the loss function. Several questions are of interest when optimal control techniques are applied to analyze and compare econometric models. 
When the optimal solution deviates so much from the historical trend, would the model remain to be valid? If not, how should the model be changed? Or should we keep the model and pull the solution closer to the trend by adding extra terms in the loss function? In fact, an important use of optimal control is to reveal the properties of an econometric model under systematic variations of the control variables. If we adopt a policy closer to the historical trend, such as 40.0 billion for federal nondefense purchases as compared with the optimal value of 78.3 billion, how much would we lose if the model remained valid at the optimum solution? (The answer is that the multiperiod loss would increase from the minimum 248.03 by only 12.65 , instead of 26.00 , when the two control variables equal 26.377 and 40.0, respectively.)

In this paper, we have reviewed briefly some existing tools for characterizing and comparing econometric models and described several techniques based on the theories of optimal control of deterministic and stochastic econometric models. In particular, a curve depicting the best inflation-unemployment trade-off can be used to characterize an econometric model. Furthermore, we propose the use of a quadratic function $V_{1 \mathrm{~A}}\left(x_{1}\right)$ which measures the $T$-period expected loss based on a model A if $x_{1}$ is the policy for period 1 while the policies for the remaining periods from 2 to $T$ will be optimal. This function is simple; it has nine parameters as illustrated by (4.2) when there are three control variables, and five parameters as illustrated by (4.4) when there are two control variables no matter how complicated model $A$ is. It captures the essential information concerning the response of the model to the policy variables and can be used to characterize a model from the viewpoint of policy analysis and formulation.

\section{ACKNOWLEDGMENTS}

I would like to thank Ettie H. Butters for excellent programming assistance and J. Kmenta, J. Ramsey, and a referee for helpful comments and to acknowledge financial support from the National Science Foundation.

\section{REFERENCES}

Anderson, L. C., \& Carlson, R. M. An econometric analysis of the relation of monetary variables to the behavior of prices and unemployment. In O. Eckstein (Ed.), The econometrics of price determination. Washington, D.C.: Board of Governors of The Federal Reserve System, 1972. Pp. 166-183.

Ando, A., \& Palash, Carl. Some stabilization problems of 1971-75, with an application of optimal control algorithms. American Economic Review, 1976, 66(2), 346-348.

Bodkin, R. D. Wage and price formation in selected Canadian econometric models. In O. Eckstein (Ed.), The econometrics of price determination. Washington, D.C.: Board of Governors of The Federal Reserve System, 1972. Pp. 369-385. 
Chow, G. C. Analysis and control of dynamic economic systems. New York: Wiley, 1975.

Chow, G. C., An approach to the feedback control of nonlinear econometric systems. Annals of Economic and Social Measurement, 1976, 5(3), 297-310.

Chow, G. C., Evaluation of macroeconomic policies by stochastic control techniques. International Economic Review, 1978, 19(2), 311-320.

Chow, G. C., Evaluation of econometric models by decomposition and aggregation. In J. Kmenta and J. Ramsey (Eds.), Methodology of macroeconomic models. Amsterdam: North-Holland Publ. 1980, in press.

Chow, G. C., \& Megdal, S. B. The control of large scale econometric systems. IEEE Transactions on Automatic Control, 1978, AC-23(2), 344-349.(a)

Chow, G. C., \& Megdal, S. B. An econometric definition of the inflation-unemployment tradeoff. American Economic Review, 1978, 68(3), 446-453.(b)

Craine, R., Havenner, A., \& Tinsley, P. Optimal macroeconomic control policies. Annals of Economic and Social Measurement, 1976, 5(2), 191-204.

de Menil, G., \& Enzler, J. J. Prices and wages in the FR-MIT-Penn econometric model. In O. Eckstein (Ed.), The econometrics of price determination. Washington, D.C.: Board of Governors of The Federal Reserve System, 1972. Pp. 277-308.

Fair, R. C. On the solution of optimal control problems as maximization problems. Annals of Economic and Social Measurement, 1974, 3(1), 135-154.

Hirsch, A. A. Price simulations with the OBE econometric model. In O. Eckstein (Ed.), The Econometrics of price determination, Washington, D.C.: Board of Governors of The Federal Reserve System, 1972. Pp. 237-276.

Hirsch, A. A., Hymans, S. H., \& Shapiro, H. Econometric review of alternative fiscal and monetary policies, 1971-75. Review of Economics and Statistics, 1978, LX(3), 334-345.

Holbrook, R. S. A practical method for controlling a large nonlinear stochastic system. Annals of Economic and Social Measurment, 1974, 3(1), 155-176.

Hymans, S. H. Prices and price behavior in three U.S. econometric models. In O. Eckstein (Ed.), The econometrics of price determination. Washington, D.C.: Board of Governors of The Federal Reserve System, 1972. Pp. 309-324.

Hymans, S. H., \& Shapiro, H. The Michigan quarterly econometric model of the U.S. economy. The Economic Outlook for 1973, Ann Arbor, Michigan: The University of Michigan, 1973, Pp. 113-155.

Kalchbrenner, J. H., \& Tinsley, P. A. On the use of feedback control in the design of aggregate monetary policy. American Economic Review, 1976, 66(2), 349-355.

Norman, A., Norman M., \& Palash, C. On the computation of deterministic optimal macroeconomic policy, Research Paper No. 7507, Federal Reserve Bank of New York, 1975. 